\title{
Sociocultural factors associated with attitudes toward suicide in Australia
}

\section{Tammie T. Money \& Philip J. Batterham}

To cite this article: Tammie T. Money \& Philip J. Batterham (2019): Sociocultural factors associated with attitudes toward suicide in Australia, Death Studies, DOI: 10.1080/07481187.2019.1626943

To link to this article: https://doi.org/10.1080/07481187.2019.1626943

\section{Published online: 13 Jun 2019.}

Submit your article to this journal

Џll Article views: 100

Q View related articles $\leftarrow$

View Crossmark data ð 


\title{
Sociocultural factors associated with attitudes toward suicide in Australia
}

\author{
Tammie T. Money a and Philip J. Batterham ${ }^{\mathrm{b}}$ \\ ${ }^{a}$ Medical School, The Australian National University, Canberra, Australia; ${ }^{b}$ Centre for Mental Health Research, Research School of \\ Population Health, The Australian National University, Canberra, Australia
}

\begin{abstract}
To investigate the role of cultural and economic factors on suicide attitudes and stigma toward suicide, we recruited 230 Australian adults for an online survey, assessing demographic characteristics (including ethnicity and socioeconomic status, SES), suicide attitudes, stigma toward people who die by suicide, and public knowledge of suicide prevention (suicide literacy). Regression analyses indicated that participants affiliated with non-Anglo cultures or with higher SES had higher suicide stigma and lower suicide literacy than Anglo participants and those in lower SES areas. Increased stigma in minority cultural groups may impact help-seeking behaviors that protect against suicide.
\end{abstract}

Pervasive stigmatizing attitudes toward people who seek help for suicidal thoughts or behaviors significantly increases the risk of suicide (World Health Organization, 2014). Stigma may also have far-reaching effects on the mental, physical and social wellbeing of those who experience it (Link \& Phelan, 2006). Given that suicide stigma may increase the risk of suicide in those who experience it, it is vital to understand the extent of suicide stigma in the community and the factors associated with it.

Few studies have investigated suicide stigma as an outcome, prior to the development of the Stigma of Suicide Scale (Batterham, Calear, \& Christensen, 2013b). In an Australia community sample, 25\% of those surveyed considered people who die by suicide to be "weak," "reckless," or "selfish" (Batterham et al., 2013b). Furthermore, respondents who spoke a language other than English at home were more likely to hold stigmatizing attitudes than those who only spoke English (Batterham, Calear, \& Christensen, 2013a).

The association between greater suicide stigmatizing attitudes in people who speak a language other than English may point toward cultural differences between English speakers and non-English speakers in relation to suicide. Certainly, suicide rates vary considerably between countries and between broad cultural groups (World Health Organization, 2018). The variation in suicide rate by country may reflect intrinsic factors including cultural or religious beliefs and genetic vulnerability or extrinsic factors such as economic stress, war and conflict, poverty, and environmental disasters. The impact of culture on both suicide rates and stigma toward suicide is demonstrated in numerous studies comparing countries that are more permissive of suicide with those that are not (Eskin, Voracek, Stieger, \& Altinyazar, 2011). This relationship may be mediated by differences in public knowledge of suicide, termed suicide literacy (Batterham et al., 2013b). To better inform suicide prevention programs, it is therefore important to understand the extent of suicide stigma, whether cultural factors influence stigma, and whether the effects of culture on stigma may be attributable to differences in suicide literacy.

One driver of suicide stigma may be lack of education about suicide, evidenced by the association between lower educational attainment and greater suicide stigma (Park, Kim, Cho, \& Lee, 2015). This association may reflect a broader influence of socioeconomic status (SES) on stigma, although this relationship has yet to be examined in detail. SES refers to the economic and social position of an individual relative to others and may be variable across time. There is evidence that low SES may be directly associated with suicide risk (Branas et al., 2015; Nordt, Warnke, Seifritz, \& Kawohl, 2015), with higher rates of suicide attempts among people with less education and unemployed (Kim, Kim, Choi, Lee, \& Park, 2016). Given the definitional challenges of SES, previous studies have used proxies such as income, 
education, and unemployment; however, these typically produce heterogeneous results and may not reflect the relative poverty or deprivation within a population (Rehkopf \& Buka, 2006). Population ranking SES metrics incorporate the number of households with a low income, few qualifications, and low skill occupations in a given geographic area. Individual and area-level socio-economic deprivation may have differential effects on stigma and suicidal attitudes, reflecting the mechanisms underlying these putative relationships.

An individual's tendency to consider suicide as a solution to difficult situations (predicaments) is an important consideration when examining factors associated with suicide stigma. Predicaments are defined as a set of circumstances from which there is limited escape, increasing the likelihood of the individual choosing suicide, irrespective of mental illness (Pridmore, 2015). Strong cultural motivators such as shame may play a role in shaping an individual's risk for suicide in response to a difficult life event (Lester, 2007). However, it remains unclear whether attitudes toward suicide in relation to specific predicaments are distinct from suicide stigma. Determining the effects of culture and SES on both suicide stigma and suicide in response to predicaments may provide insights into the role of social determinants in suicide.

This study aims to explore the contribution of culture and SES on both suicide stigma and suicide attitudes in response to predicaments. We also examine the role of culture and SES on suicide literacy (public knowledge about suicide) to determine whether any differences in attitudes may be related to gaps in knowledge. Finally, we examine whether cultural differences in stigma are explained by differences in suicide literacy. Our hypothesis is that there will be greater stigmatization of suicide in Australian people with non-Western cultural affiliation and people with lower SES, independent of suicide literacy.

\section{Method}

\section{Participants}

A total of 230 participants completed an online survey assessing demographic characteristics (including ethnicity and socioeconomic status, SES), suicide stigma, suicide attitudes, suicide literacy, and suicidal thoughts and behaviors. The sample was mainly women, with $49 \%$ aged between 18 and 34 years, typically earning under $\$ 39,000$ per year (64\%), with most holding a university degree (67\%) and living in cities or towns with population fewer than 500,000 (65\%). All participants completed all scales, so no missing data were present. The sample had elevated levels of suicidal thoughts and behaviors, with $32 \%$ reporting suicidal ideation in the past year, $20 \%$ a plan and $9 \%$ an attempt.

\section{Procedure}

We used Facebook advertising to recruit participants who were over 18 years and living in Australia. We targeted posts on a Facebook page titled "The effect of social factors on suicide" to our study population using paid advertising to promote the study on the news feeds of Facebook users in the community. The posts included a direct link to the online survey. We targeted the advertising to Australian adults on Facebook (approximately $75 \%$ of the total Australian adult population), with a proportion of the advertising targeted to individuals who listed languages other than English in their profiles to purposive oversample ethnically diverse individuals.

Facebook advertising included a brief description of the research and a link to the online survey. The online survey was preceded by a participant information sheet with detailed information about the research, the potentially distressing nature of the questions, the estimated time needed for completion of the survey, contact details for both the ethics committee and researchers, and referral information for helpline and counseling services. Participants provided consent by clicking "yes" to being over 18 years of age and residing in Australia and "yes" to agree to participate in the survey. Participation was anonymous with no financial incentive offered. Participants completed the survey online in a single sitting and responses were recorded anonymously. At the completion of the survey, participants received contact details for helpline and counseling services. Survey data were collected using the Qualtrics survey software. The Human Research Ethics Committee at [placeholder] approved the ethical conduct of the project.

\section{Measures}

We measured suicide stigma with the Stigma of Suicide Scale Short form (SOSS-SF), a 16-item scale that assesses attitudes toward a "typical" person who died by suicide using adjectives (Batterham et al., 2013b). The questions apply to a nonspecific person who dies by suicide and has a 5-point Likert scale (strongly disagree, disagree, neutral, agree, and strongly agree). The introductory wording to the SOSS is "Using the scale below, please rate how much you 
agree with the descriptions of people who take their own lives (suicide). In general, people who suicide are ..." and descriptors are listed in alphabetical order. The SOSS-SF comprises three factors: Stigma (reflecting negative attitudes toward people who have died by suicide), Isolation/Depression (reflecting attribution of suicide to isolation or depression), and Normalisation/ Glorification (reflecting a viewpoint that suicide indicates strength or bravery). Means for the Stigma, Isolation/Depression and Normalisation/Glorification subscales in this sample were $1.84(\mathrm{SD}=0.98), 4.10$ $(\mathrm{SD}=0.85)$ and $2.56(\mathrm{SD}=0.97)$, respectively. The scale has previously been shown to have strong psychometric properties in similar samples (Batterham et al., 2013b; Williams, Cero, Gauthier, \& Witte, 2018). The focus of the current study was only on the stigma subscale, which comprised eight items with high internal consistency (Cronbach $\mathrm{a}=0.88$ ).

The Predicament Questionnaire (PQ), a 32-item questionnaire of suicide attitudes that presents fictionalized adverse predicaments in which an individual might experience suicidal thoughts (Shahtahmasebi, Varbanov, Aleksandrov, \& Pridmore, 2016). Respondents rate each situation based on the likelihood that it would induce suicidal thoughts (no, slight, moderate, strong). An example of an item is "Person E suffers spinal injuries and will be confined to a wheelchair for life. Would person $\mathrm{E}$ have suicidal thoughts?" Total suicidal attitude (TSA) was calculated for each participant as the mean of the responses across all predicaments (where $n o=1$ and strong $=4$ ) for a total of between 1 (low TSA) and 4 (very high TSA). The PQ showed good internal validity (Cronbach $a=0.86$ in the current sample) and factor analysis has previously demonstrated unidimensionality in a sample predominantly consisting of Englishspeaking young adults (15-24 years) (Shahtahmasebi et al., 2016). The mean TSA was 2.32 in the sample $(\mathrm{SD}=1.04)$.

We assessed suicide literacy using the LOSS, which includes 12 true/false items and has previously been validated using item response theory as the responses are either correct or incorrect (Batterham et al., 2013a; Calear, Batterham, \& Christensen, 2014). An example of an item is "people who have thoughts about suicide should not tell others about it" and participants select "true," "false," or "I don't know." Correct responses were scored 1 while incorrect or "I don't know" responses were scored 0 . Literacy scores are the sum of correct items, with higher scores indicating higher suicide literacy. The mean LOSS score was 9.06 in this sample ( $\mathrm{SD}=1.92)$.
Ethnicity: For ethnicity, we used the Australian Bureau of Statistics Australian Standard of Classification of Cultural and Ethnic Groups, 2016 to stratify our sample into two ethnic groups. We classified the group henceforth referred to as "Anglo" based on the broad group "Oceanian," which includes people identifying their ethnicity as from Australia, New Zealand, Britain, and Ireland, as these are broadly based on a common ancestry. We classified participants who identified any other ethnicity or who spoke a language other than English at home as "non-Anglo."

Socio-economic status: We used self-reported income brackets $(<\$ 12,000, \$ 13,000-\$ 24,000$, $\$ 25,000-\$ 39,000, \quad \$ 40,000-\$ 59,000, \quad \$ 60,000-\$ 79,000$, $\$ 80,000-99,000$, or $>\$ 100,000)$ and employment status to assess individual SES. Employment was classified as full-time (30 or more hours per week), parttime (less than 30 hours per week), casual (on-demand with varying hours), retired, or unemployed. Arealevel SES was determined from participants' postcodes using the Australian Bureau of Statistics Index of Relative Socio-economic Disadvantage (IRSD). Using information about the economic and social conditions of people residing in each area, the IRSD ranks postcodes into 10 index scores; where 1 indicates most disadvantaged and 10 indicates least disadvantaged. IRSD score was included as a continuous variable in regression analyses although summarized into IRSD bands for the purpose of describing the sample. The majority of the sample lived in a neighborhood with an IRSD band of $8-10(54 \%)$ although $24 \%$ lived in a disadvantaged neighborhood (band 1-4).

Suicidal thoughts and behaviors: Suicidal thoughts, plans, and attempts were assessed using the four suicide items from the Youth Risk Behavior survey (Brener et al., 2002). These were scored yes-no and the number of suicide attempts were grouped from 1 to 5 , with 1 being no suicide attempts and 5 being 6 or more suicide attempts.

\section{Results}

Most participants $(n=186,81 \%)$ were classified as "Anglo," with the remainder $(n=44,19 \%)$ nonAnglo. An independent samples $t$-test demonstrated that there was a significant difference in suicide stigma between ethnicities $(t=-2.88, d f=228$, $p=0.004$, Cohen's $d=0.47$ ), with higher scores for the non-Anglo participants (mean \pm SD: $2.11 \pm 0.76$ ) compared to Anglo participants (mean \pm SD: $1.77 \pm 0.68)$. Total scores for predicaments were not 
significantly different between ethnicities $(t=-1.92$, $d f=228, p=0.056$ ). Suicide literacy was significantly different between ethnicities $(t=-3.19, d f=228$, $p=0.002, d=0.50)$, with lower scores for non-Anglo participants (mean \pm SD: $8.25 \pm 2.18$ ) compared to Anglo participants (mean \pm SD: $9.26 \pm 1.81$ ) .

People from different SES categories (based on IRSD bands within postcodes) had significantly different suicide stigma based on SOSS-stigma scores $(t=2.313, d f=228, p=0.022)$, with higher SES associated with greater stigma. There were, however, no significant differences between SES categories for predicament scores $(t=-0.245, d f=228, p=0.807)$ or suicide literacy scores $(t=-0.922, d f=228$, $p=0.358)$. There was no significant difference between income brackets for suicide stigma scores $(F=1.266, d f=7,222, p=0.268)$, predicament scores $(F=1.500, d f=7,222, p=0.168)$ or suicide literacy scores $(F=0.831, d f=7,222, p=0.563)$. Finally, there was no significant effect of employment status on suicide stigma scores $(F=0.510, d f=4,225, p=0.728)$, predicament scores $(F=1.214, d f=4,225, p=0.306)$. There was, however, a significant effect of employment status on suicide literacy scores $(F=1.011$, $d f=4,225, p=0.403)$, with retired and unemployed participants having lower suicide literacy than those in full-time employment.

All sociodemographic factors and indicators of suicidal thoughts or behaviors were included in linear regression models for each of the three outcome measures. The models for suicide stigma $\left(R^{2}=.18\right.$; $F=1.62, d f=27,201, p=0.034)$, predicaments $\left(R^{2}=\right.$ $0.18 ; F=1.58, d f=27,201, p=0.041)$, and suicide literacy $\left(R^{2}=0.190 ; F=1.75, d f=27,201, p=0.016\right)$ explained significant variance in these outcomes. Ethnicity was significantly associated with suicide stigma and suicide literacy scores: non-Anglo participants scored 0.3 points higher (out of 5) on the SOSS-stigma measure of suicide stigma and one point lower (out of 12) on the LOSS measure of suicide literacy. SES, as measured by IRSD band, was also significantly associated with these two outcomes, with a 0.05 increase in stigma scores and a decrease of 0.1 points on literacy scores per increase in IRSD band. There was also an independent effect of personal income bracket on suicide stigma, with those earning between $\$ 13,000$ and $\$ 39,000$ reporting higher levels of stigma than those earning less. No other sociodemographic variables had a significant effect on any of the outcomes. The models are reported in Table 1.

To further explore whether the effect of ethnicity on suicide stigma could be explained by suicide literacy, a sensitivity analysis re-estimated the suicide stigma model including ethnicity, income, IRSD band, and suicide literacy. In this model, the effects of ethnicity $(t=2.12, d f=205, \quad p=0.036), \quad$ SES $(t=2.01$, $d f=205, p=0.046)$ and suicide literacy $(t=-2.32$, $d f=205, p=0.021)$ all remained significant. Similarly, when suicide stigma was added to the suicide literacy model, the effects of ethnicity $(t=-2.87, d f=205$, $p=0.005)$ and suicide stigma $(t=-2.32, d f=205$, $p=0.021)$ remained significant although the effect of SES was attenuated and non-significant $(t=-0.36$, $d f=205, p=0.723$ ).

\section{Discussion}

In support of the hypothesis that ethnicity would have a significant effect on stigma toward suicidal people, the results revealed that non-Anglo participants had significantly higher scores for stigma toward suicide than Anglo participants in both univariate and multivariate analyses. This result is consistent with a previous study showing that participants speaking a language other than English at home had greater suicide stigma (Batterham et al., 2013b), which was attributed to potential cultural differences. Literacy of suicide was also significantly different between broad ethnic categories, suggesting that the increase in stigma in non-Anglo participants may be partially explained by a lack of literacy. However, when suicide literacy was included in the suicide stigma model (or vice-versa), the effect of ethnicity remained significant, suggesting an independent relationship. Overall these results support previous research that certain cultures have more stigmatizing attitudes toward suicide than other cultures (Eskin et al., 2011). Our study has demonstrated that in Australia, non-Anglo cultural groups have more stigmatizing attitudes.

There was a significant effect of the populationlevel SES measure on stigma and literacy of suicide. However, the effect was contrary to hypotheses, with higher SES associated with more stigma and lower literacy. These findings are inconsistent with findings around stigma toward mental illness (Evans-Lacko, Henderson, \& Thornicroft, 2013; Holman, 2015; von dem Knesebeck et al., 2013) and findings indicating associations between suicide deaths and low SES (Kim, Park, \& Yoo, 2015). It is possible that higher exposure to suicide in low SES neighborhoods (Kim et al., 2015) underlies the observed relationship, as exposure to suicide is closely related to reduced stigma and increased literacy (Batterham et al., 2013a). Alternatively, it may be the case that suicide 
Table 1. Linear regression models of suicide stigma, suicide literacy, and suicide attitudes.

\begin{tabular}{|c|c|c|c|c|c|c|}
\hline & \multicolumn{2}{|c|}{ Suicide stigma } & \multicolumn{2}{|c|}{ Suicide literacy } & \multicolumn{2}{|c|}{ Suicide attitudes } \\
\hline & Estimate & $p$ & Estimate & $p$ & Estimate & $p$ \\
\hline Intercept & 0.903 & 0.047 & 9.817 & $<0.001$ & 2.247 & $<0.001$ \\
\hline Gender & & 0.515 & & 0.857 & & 0.886 \\
\hline Women & -0.073 & 0.534 & -0.074 & 0.815 & -0.030 & 0.623 \\
\hline Other & -0.345 & 0.269 & 0.352 & 0.676 & -0.019 & 0.906 \\
\hline Men & 0.000 & & 0.000 & & 0.000 & \\
\hline Age group & & 0.085 & & 0.620 & & 0.507 \\
\hline $18-24$ years & 0.791 & 0.021 & 0.685 & 0.457 & -0.022 & 0.900 \\
\hline $25-34$ years & 0.642 & 0.056 & 1.073 & 0.238 & -0.136 & 0.440 \\
\hline $35-44$ years & 0.420 & 0.223 & 1.231 & 0.187 & -0.036 & 0.840 \\
\hline $45-54$ years & 0.549 & 0.102 & 0.704 & 0.439 & -0.065 & 0.712 \\
\hline $55-64$ years & 0.286 & 0.350 & 0.836 & 0.313 & 0.052 & 0.746 \\
\hline 65 years and above & 0.000 & & 0.000 & & 0.000 & \\
\hline Income & & 0.030 & & 0.109 & & 0.187 \\
\hline$\$ 13,000-\$ 24,000$ & 0.290 & 0.034 & -0.234 & 0.525 & 0.057 & 0.424 \\
\hline$\$ 25,000-\$ 39,000$ & 0.469 & 0.009 & -0.693 & 0.151 & 0.101 & 0.279 \\
\hline$\$ 40,000-\$ 59,000$ & 0.035 & 0.874 & -0.144 & 0.808 & 0.199 & 0.084 \\
\hline$\$ 60,000-\$ 79,000$ & -0.231 & 0.272 & 0.030 & 0.958 & 0.135 & 0.223 \\
\hline$\$ 80,000-\$ 99,000$ & 0.238 & 0.288 & -1.391 & 0.023 & -0.130 & 0.269 \\
\hline Over $\$ 100,000$ & 0.098 & 0.673 & -0.213 & 0.734 & -0.030 & 0.807 \\
\hline Prefer not to say & 0.449 & 0.161 & 1.301 & 0.134 & 0.089 & 0.595 \\
\hline Less than $\$ 12,000$ & 0.000 & & 0.000 & & 0.000 & \\
\hline Education: bachelors vs less & 0.086 & 0.421 & -0.514 & 0.076 & -0.006 & 0.918 \\
\hline Employment status & & 0.065 & & 0.172 & & 0.362 \\
\hline Employed, Part time & -0.202 & 0.266 & -0.394 & 0.422 & 0.011 & 0.905 \\
\hline Employed, Casual & -0.372 & 0.041 & 0.292 & 0.553 & 0.004 & 0.969 \\
\hline Retired & 0.325 & 0.280 & -0.925 & 0.257 & -0.253 & 0.110 \\
\hline Unemployed & -0.075 & 0.660 & -0.522 & 0.261 & 0.067 & 0.455 \\
\hline Employed, Full time & 0.000 & & 0.000 & & 0.000 & \\
\hline Ethnicity: non-Anglo vs Anglo & 0.275 & 0.025 & -1.035 & 0.002 & -0.122 & 0.059 \\
\hline Location & & 0.186 & & 0.845 & & 0.798 \\
\hline City $100,000-500,000$ residents & -0.102 & 0.393 & 0.225 & 0.488 & 0.005 & 0.938 \\
\hline City $10,000-100,000$ residents & -0.132 & 0.423 & 0.267 & 0.549 & 0.044 & 0.612 \\
\hline Village/rural & 0.226 & 0.153 & -0.032 & 0.941 & 0.081 & 0.332 \\
\hline City $>500,000$ residents & 0.000 & & 0.000 & & 0.000 & \\
\hline SES band & 0.045 & 0.026 & -0.112 & 0.041 & 0.006 & 0.549 \\
\hline Suicide ideation & -0.105 & 0.495 & 0.311 & 0.455 & 0.108 & 0.182 \\
\hline Suicide plan & -0.084 & 0.661 & -0.390 & 0.453 & 0.185 & 0.067 \\
\hline Suicide attempt & -0.087 & 0.667 & -0.467 & 0.392 & -0.166 & 0.117 \\
\hline
\end{tabular}

stigma manifests differently to the stigma of mental illness. Nevertheless, the effect was small and further investigation of this potentially complex relationship is required. Interestingly, the individual SES measures of income and employment showed no significant relationship with suicide attitudes or knowledge in the regression analyses, suggesting that community-level factors may have a stronger impact on attitudes toward suicide than individual factors. The findings may also have been influenced by challenges in the accurate measurement of individual SES (Shavers, 2007).

The challenges of sampling ethnically diverse Australians limited our ability to identify specific ethnic subgroups with high levels of stigma. Further research is needed to examine potential mechanisms by which culture may influence stigma. The relationship between stigma and help-seeking behavior is crucial to elucidating the impact of this finding on suicide prevention outcomes. The WHO suggests that globally, stigma decreases help-seeking behaviors (World Health Organization, 2014) but in studies of specific cultural groups, stigma actually increased the likelihood that a suicidal person would receive informal social support in order to prevent suicide (Eskin et al., 2011). While informal supports may be beneficial, professionals are best equipped to provide evidence-based care. Further investigation of the patterns of help seeking in different cultural and ethnic groups might yield specific culturally-appropriate approaches to supporting suicidal individuals. Similar to other cohorts, a history of suicidal ideation or behavior had no direct relationship with suicide stigma or suicide literacy (Batterham et al., 2013a).

Contrary to our hypothesis, there was no significant difference between ethnicities for suicidal attitudes in response to specific predicaments. It suggests that the predicaments within the questionnaire induce 
comparable likelihood of choosing suicide as a response regardless of cultural affiliation and that the level of suicidal attitudes is similar between cultures. The findings also suggest that suicidal attitudes in response to specific predicaments are distinct from suicide stigma, which reflects personal stigma in response to a hypothetical suicidal individual. The PQ measure may be capturing perceived attitudes around the permissibility of suicide within the community, rather than personal attitudes toward suicidal individuals. The findings suggest that personal stigma might be a more pertinent target for suicide prevention programs than attitudes related to specific predicaments. Further studies using a greater representation of cultures is needed, but the present findings suggest that either the sample was too culturally homogeneous to find subtle differences in PQ scores or that broad attitudes toward suicidal people are distinct from attitudes toward individuals in specific predicaments.

The small sample size limited our ability to include specific cultural groups in order to more accurately measure the effect of different cultures on suicidal attitudes and stigma. However, the effect of ethnicity on stigma between Anglo and non-Anglo participants was clearly demonstrated and warrants replication in a larger cohort with greater numbers of participants representing ethnic groups in Australia. Further studies may reveal particular cultures with considerably elevated stigma, which accounts for the difference in the broader cultural group or a general increase in stigma across many non-Anglo cultures. Qualitative and ethnographic research in specific cultural groups might prove valuable. Whilst self-report surveys can be limited by desirability bias, our use of an anonymous online survey allowed participants the freedom of honesty and reduced desirability bias compared to a more direct method of survey (Bowling, 2005).

This study suggests that stigma toward people who die by suicide is higher in non-Anglo Australians and those in higher SES communities. Similarly, suicide literacy appears to be higher in Anglo Australians and people living in lower SES communities. In addition, the effect of culture on suicide stigma was not accounted for by suicide literacy. Suicidal attitudes in response to a set of predicaments were relatively similar across broad cultural groups and were not affected by SES. These results suggest that suicide prevention interventions may need targeting and tailoring for people who identify with minority ethnic groups, as such individuals may be vulnerable and particularly reluctant to seek help.

\section{Disclosure statement}

No potential conflict of interest was reported by the authors.

\section{Funding}

PJB is supported by NHMRC Fellowship [1158707].

\section{References}

Batterham, P. J., Calear, A. L., \& Christensen, H. (2013a). Correlates of suicide stigma and suicide literacy in the community. Suicide and Life-Threatening Behavior, 43(4), 406-417. doi:10.1111/sltb.12026

Batterham, P. J., Calear, A. L., \& Christensen, H. (2013b). The stigma of suicide scale. Psychometric properties and correlates of the stigma of suicide. Crisis, 34(1), 13-21. doi:10.1027/0227-5910/a000156

Bowling, A. (2005). Mode of questionnaire administration can have serious effects on data quality. Journal of Public Health ( Health), 27(3), 281-291. doi:10.1093/pubmed/ fdi031

Branas, C. C., Kastanaki, A. E., Michalodimitrakis, M., Tzougas, J., Kranioti, E. F., Theodorakis, P. N., ... Wiebe, D. J. (2015). The impact of economic austerity and prosperity events on suicide in Greece: A 30-year interrupted time-series analysis. BMJ Open, 5(1), e005619. doi:10.1136/bmjopen-2014-005619

Brener, N. D., Kann, L., McManus, T., Kinchen, S. A., Sundberg, E. C., \& Ross, J. G. (2002). Reliability of the 1999 youth risk behavior survey questionnaire. Journal of Adolescent Health, 31(4), 336-342. doi:10.1016/S1054139X(02)00339-7

Calear, A. L., Batterham, P. J., \& Christensen, H. (2014). Predictors of help-seeking for suicidal ideation in the community: Risks and opportunities for public suicide prevention campaigns. Psychiatry Research, 219(3), 525-530. doi:10.1016/j.psychres.2014.06.027

Eskin, M., Voracek, M., Stieger, S., \& Altinyazar, V. (2011). A cross-cultural investigation of suicidal behavior and attitudes in Austrian and Turkish medical students. Social Psychiatry and Psychiatric Epidemiology, 46(9), 813-823. doi:10.1007/s00127-010-0254-7

Evans-Lacko, S., Henderson, C., \& Thornicroft, G. (2013). Public knowledge, attitudes and behaviour regarding people with mental illness in England 2009-2012. British Journal of Psychiatry Supplement, 55, S51-S57. doi:10.1192/bjp.bp.112.112979

Holman, D. (2015). Exploring the relationship between social class, mental illness stigma and mental health literacy using British national survey data. Health: An Interdisciplinary Journal for the Social Study of Health, Illness and Medicine), 19(4), 413-429. doi:10.1177/ 1363459314554316

Kim, J., Kim, J. M., Choi, Y., Lee, T. H., \& Park, E. C. (2016). Effect of socioeconomic status on the linkage between suicidal ideation and suicide attempts. Suicide and Life-Threatening Behavior, 46(5), 588-597. doi: $10.1111 /$ sltb. 12242 
Kim, J., Park, E. C., \& Yoo, K. B. (2015). Effects of the gap between socioeconomic status and perceived social class on suicidal ideation: Unique perspectives using a longitudinal analysis. Archives of Gerontology and Geriatrics, 61(3), 384-391. doi:10.1016/j.archger.2015.06.002

Lester, D. (2007). The causes of changing suicide rates. Psychological Reports, 100(3_suppl), 1140. doi:10.2466/ pr0.100.4.1140-1140

Link, B. G., \& Phelan, J. C. (2006). Stigma and its public health implications. Lancet, 367(9509), 528-529. doi:10.1016/S0140-6736(06)68184-1

Nordt, C., Warnke, I., Seifritz, E., \& Kawohl, W. (2015). Modelling suicide and unemployment: A longitudinal analysis covering 63 countries, 2000-11. Lancet Psychiatry, 2(3), 239-245. doi:10.1016/S2215-0366(14) 00118-7

Park, S., Kim, M. J., Cho, M. J., \& Lee, J. Y. (2015). Factors affecting stigma toward suicide and depression: A Korean nationwide study. International Journal of Social Psychiatry, 61(8), 811-817. doi:10.1177/002076401 5597015

Pridmore, S. (2015). Mental disorder and suicide: A faulty connection. Australian \& New Zealand Journal of Psychiatry, 49(1), 18-20. doi:10.1177/0004867414548904

Rehkopf, D. H., \& Buka, S. L. (2006). The association between suicide and the socio-economic characteristics of geographical areas: A systematic review. Psychological
Medicine, 36(2), 145-157. doi:10.1017/S003329170 500588X

Shahtahmasebi, S., Varbanov, A., Aleksandrov, I., \& Pridmore, S. (2016). Exploring social attitudes to suicide using a predicament questionnaire. Open Journal of Social Sciences, 04(12), 58-71. doi:10.4236/jss.2016.412006

Shavers, V. L. (2007). Measurement of socioeconomic status in health disparities research. Journal of the National Medical Association, 99(9), 1013-1023.

von Dem Knesebeck, O., Mnich, E., Daubmann, A., Wegscheider, K., Angermeyer, M. C., Lambert, M., ... Kofahl, C. (2013). Socioeconomic status and beliefs about depression, schizophrenia and eating disorders. Social Psychiatry and Psychiatric Epidemiology, 48(5), 775-782. doi:10.1007/s00127-012-0599-1

Williams, C. L., Cero, I., Gauthier, J. M., \& Witte, T. K. (2018). Examination of the latent factor structure and construct validity of the stigma of suicide scale-short form. Death Studies, 42(10), 616-626. doi:10.1080/ 07481187.2017.1416431

World Health Organization. (2014). Preventing suicide: A global imperative. Retrieved from https://www.who.int/ mental_health/suicide-prevention/world_report_2014/en/

World Health Organization. (2018). Global Health Estimates 2016: Deaths by cause, age, sex, by country and by region, 2000-2016. Geneva: World Health Organization. Retrieved from https://www.who.int/mental_health/suicide-prevention/en/ 